ARTICLE

DOI: $10.1038 /$ s41467-018-07265-z

\title{
Metastable silica high pressure polymorphs as structural proxies of deep Earth silicate melts
}

\author{
E. Bykova ${ }^{1,2}$, M. Bykov (1) 2,3, A. Černok (10 2,9, J. Tidholm4, S.I. Simak (1) 4, O. Hellman (D) 4,5, M.P. Belov ${ }^{3}$, \\ I.A. Abrikosov"4, H.-P. Liermann (1) 1, M. Hanfland ${ }^{6}$, V.B. Prakapenka7 , C. Prescher 7,10, \\ N. Dubrovinskaia ${ }^{8} \&$ L. Dubrovinsky ${ }^{2}$
}

Modelling of processes involving deep Earth liquids requires information on their structures and compression mechanisms. However, knowledge of the local structures of silicates and silica $\left(\mathrm{SiO}_{2}\right)$ melts at deep mantle conditions and of their densification mechanisms is still limited. Here we report the synthesis and characterization of metastable high-pressure silica phases, coesite-IV and coesite- $\mathrm{V}$, using in situ single-crystal $\mathrm{X}$-ray diffraction and ab initio simulations. Their crystal structures are drastically different from any previously considered models, but explain well features of pair-distribution functions of highly densified silica glass and molten basalt at high pressure. Built of four, five-, and six-coordinated silicon, coesite-IV and coesite- $\mathrm{V}$ contain $\mathrm{SiO}_{6}$ octahedra, which, at odds with $3^{\text {rd }}$ Pauling's rule, are connected through common faces. Our results suggest that possible silicate liquids in Earth's lower mantle may have complex structures making them more compressible than previously supposed.

\footnotetext{
${ }^{1}$ Photon Sciences, Deutsches Elektronen-Synchrotron (DESY), Notkestraße 85, 22607 Hamburg, Germany. ${ }^{2}$ Bayerisches Geoinstitut, University of Bayreuth, Universitätsstraße 30, 95440 Bayreuth, Germany. ${ }^{3}$ Materials Modeling and Development Laboratory, National University of Science and Technology 'MISIS', Leninsky Avenue 4, 119049 Moscow, Russia. ${ }^{4}$ Department of Physics, Chemistry and Biology, Linköping University, SE-581 83 Linköping, Sweden.

${ }^{5}$ Department of Applied Physics and Materials Science, California Institute of Technology, 1200 East California Boulevard, Pasadena, California 91125, USA. ${ }^{6}$ European Synchrotron Radiation Facility (ESRF), 6 Rue Jules Horowitz, 38000 Grenoble, France. ${ }^{7}$ Center for Advanced Radiation Sources, University of Chicago, 5640 South Ellis Avenue, Chicago, Illinois 60637, USA. ${ }^{8}$ Material Physics and Technology at Extreme Conditions, Laboratory of Crystallography, University of Bayreuth, Universitätsstraße 30, 95440 Bayreuth, Germany. ${ }^{9}$ Present address: School of Physical Sciences, The Open University, Walton Hall, Milton Keynes MK7 6AA, UK. ${ }^{10}$ Present address: Institute of Geology and Mineralogy, Universität zu Köln, Zülpicher Straße 49b, 50674 Köln, Germany. Correspondence and requests for materials should be addressed to E.B. (email: elena.bykova@desy.de)
} 
T here is compiling evidence that neutrally or negatively buoyant silicate melts are present at the base of the Earth's mantle ${ }^{1-6}$. Knowledge on physical and chemical properties of the melts is important for understanding evolution of the deep Earth interiors. The local structure of melts, which is roughly characterized by the coordination number of silicon atoms and the way how the silicon polyhedra are interconnected at certain pressure-temperature conditions, can be modeled using molecular dynamics simulations ${ }^{7,8}$, Bader's atoms-in-molecules approach $^{9}$, or studied experimentally on silica or silicate glas$\operatorname{ses}^{10-12}$. Recent experimental studies of silica glass suggest that the coordination number of silicon atoms drastically increases from 4 to 6 between 15 and $60 \mathrm{GPa}$ and then, up to $\sim 100 \mathrm{GPa}$, it is either constant ${ }^{13}$ or increases ${ }^{11}$ to 7 . However, structural models are lacking and the interpretation of observations remains ambiguous, as the existence of penta-coordinated silicon in glass remains elusive. A very convincing method to obtain a structural model of noncrystalline silica material is to compare (or fit) experimental total scattering data with the pair-distribution function (PDF) of known crystalline phase $(\mathrm{s})^{14}$. In this manner, Keen and Dove ${ }^{14}$ determined that the local structure of silica glass at ambient conditions has strong similarities with HPtridymite and $\beta$-cristobalite. So far, however, there were no crystalline phase(s) which could describe features of total scattering data of silica and/or silicate glasses at high pressure.

Coesite, a dense silica polymorph, remains a subject of intense studies at high pressures and variable temperatures. At ambient conditions, coesite has a monoclinic crystal structure (space group $C 2 / c$, further called "coesite-I") and above $20 \mathrm{GPa}$, it undergoes a phase transition with doubling of the unit cell parameter $b$ (space group $P 2_{1} / c$, "coesite-II") ${ }^{15,16}$. Like in coesiteI, all silicon atoms in coesite-II were found in $\mathrm{SiO}_{4}$ tetrahedra linked through vertexes (Fig. 1, Supplementary Fig. 1a, b). Above $31 \mathrm{GPa}$, coesite-II transforms into a phase tentatively indexed as triclinic ("coesite-III") ${ }^{16}$. On the contrary, based on a combination of X-ray diffraction and ab initio metadynamics simulation, $\mathrm{Hu}$ et al. ${ }^{17}$ have proposed a transition between 26 and $53 \mathrm{GPa}$ from coesite-I to post stishovite (i.e., built of only $\mathrm{SiO}_{6}$ octahedra) through a series of triclinic intermediate phases featuring both $\mathrm{SiO}_{4}$ tetrahedra and $\mathrm{SiO}_{6}$ octahedra. Further first-principles calculations made by Liu et al. ${ }^{18}$ suggest that post-stishovite phase should eventually transform to the one with $\alpha-\mathrm{PbO}_{2}$ structure. So far, the structures of coesite-III and other possible high-pressure polymorphs of coesite remain unknown calling for further investigations.

Here, we apply single-crystal X-ray diffraction in diamond anvil cells (see Methods) in order to study $\mathrm{SiO}_{2}$ phases, which appear on compression of coesite, and their high-pressure behavior. Several independent experiments are performed at different synchrotron radiation facilities at pressures over $70 \mathrm{GPa}$. The results are summarized in Supplementary Table 1. We show that high-pressure phases of coesite can be used as proxies of the local structure of high-pressure silica melts. The crystal structure of coesite's high-pressure phases can also give an insight into the mechanisms of silica glass densification.

\section{Results}

Crystal structure of coesite-III. In agreement with previous data, in all experiments, we observed the transformation of coesite-I to coesite-II at pressures exceeding $20 \mathrm{GPa}$ (Supplementary Figs. 1, 2). A close examination of the diffraction patterns collected just above 25 GPa revealed a set of reflections which did not belong to coesite-II (Supplementary Fig. 2), and therefore should be attributed to a different phase. Indexed as triclinic (space group $P$-1), it turned out to be coesite-III (Supplementary Tables 2-4, Supplementary Figs. 1, 2).

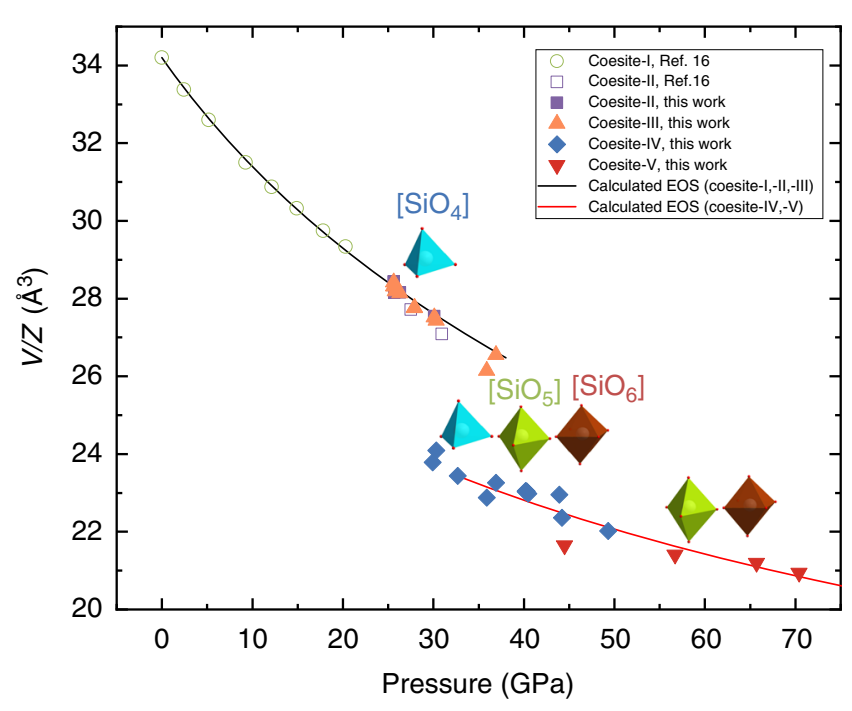

Fig. 1 The $P-V$ data of squeezed coesite. Unit cell volumes are normalized to the number of formula units $Z$. Open symbols represent literature data $^{15,16}$ (in the present work, we used samples of the same coesite which was studied in refs. $\left.{ }^{15,16}\right)$. The black solid line represents a fit of all the VPdata for coesite-I, II, and III with the Birch-Murnaghan equation of state (EOS) $\left(V_{0} / Z=34.20(1) \AA^{3}, K_{0}=103(2) \mathrm{GPa}\right.$, and $\left.K^{\prime}=3.02(15)\right)$.

Combined pressure-volume data for coesite-IV and coesite- $V$ were fitted with the second-order Birch-Murnaghan equation of state $\left(V_{32.7} / Z=23.44\right.$ (3) $\AA^{3}, K_{32.7}=254(9) \mathrm{GPa}$ ) and are shown on the graph by a solid red line. Colored polyhedra indicate the building blocks characteristic for the structures of silica polymorphs in the corresponding pressure regions. Uncertainties in the unit cell volumes are less than the symbol sizes and are therefore not shown. As seen, coesite-III and coesite-IV show significant scatter in values of the unit cell volumes that might be attributed to different deviatoric stresses in samples from different runs

We solved and refined the crystal structure of coesite-III using a dataset collected at $28 \mathrm{GPa}$ (Supplementary Figs. 1, 2, Supplementary Tables 3, 4, Supplementary Data 1). Silicon atoms in coesite-III (Supplementary Fig. 1) occupy oxygen tetrahedra linked together through common vertices. Similar to coesite-I and coesite-II, the major building blocks of the structure are fourmembered rings of $\mathrm{SiO}_{4}$ tetrahedra (Supplementary Fig. 1). Phase transitions to coesite-II and coesite-III have a minor effect on the molar volume (Fig. 1), so that the compressional behavior of all coesite phases with tetra-coordinated silicon may be described by the same Birch-Murnaghan equation of state (EOS) with $V_{0} / Z=$ $34.20(1) \AA^{3}, K_{0}=103(2) \mathrm{GPa}$, and $K^{\prime}=3.02(15)$.

Crystal structures of coesite-IV and coesite-V. On compression beyond ca. $30 \mathrm{GPa}$, a new set of reflections (Supplementary Fig. 2) manifest the presence of a new triclinic (space group $P-1$ ) phase, which we called coesite-IV (Supplementary Table 2). Upon further compression, the reflections of coesite-IV split and a new triclinic (space group $P-1$ ) phase, coesite-V emerged. Above $\sim 50$ $\mathrm{GPa}$, only coesite- $\mathrm{V}$ was found (Fig. 1). Although upon compression the quality of coesite- $\mathrm{V}$ crystals deteriorates, the phase remains crystalline (Supplementary Fig. 2) at ambient temperature to at least $70 \mathrm{GPa}$ (highest pressure achieved in this study).

The structures of coesite-IV and of coesite- $\mathrm{V}$ were solved and refined using the datasets collected at $\sim 36,40,44$, and $49 \mathrm{GPa}$ for the former (Supplementary Tables 5-8, Supplementary Data 25), and at $\sim 57 \mathrm{GPa}$ for the latter (Supplementary Data 6) (see also Supplementary Tables 2, 3, 9). For silica, known for its rich polymorphism ${ }^{17}$, the structures of coesite-IV and coesite- $\mathrm{V}$ are unusually complex (Fig. 2), but very alike. Their lattice 
parameters are very similar, and the unit cells contain $16 \mathrm{SiO}_{2}$ formula units. The structure of coesite-IV possesses tetra-, penta-, and hexa-coordinated silicon; the structure of coesite- $V$ maintains only penta- and hexa-coordinated silicon (Supplementary Fig. 3). In fact, analyzing them in detail, one can see that the structures of coesite-IV and coesite- $\mathrm{V}$ may be considered as a three-dimensional framework of face- and edge-sharing octahedra with the empty space, filled by $\mathrm{SiO}_{5}$ and $\mathrm{SiO}_{4}$ (in coesite-IV), or only $\mathrm{SiO}_{5}$ (in coesite-V) polyhedra (Fig. 2). The findings described above present three crystalchemical surprises at once: penta-coordinated silicon, face-sharing $\mathrm{SiO}_{6}$ octahedra, and the number of essentially different constituents in the structure of silica. These results are discussed below.

The size and shape of $\mathrm{SiO}_{4}$ tetrahedra in coesite-IV (Fig. 2) are comparable with those in coesite-III (Supplementary Fig. 1), although the tetrahedra in coesite-IV are more distorted in terms of the bond angle variance $(\mathrm{BAV})^{19}$. Measured in squared degrees, BAV in coesite-IV is equal to $\sim 81$, whereas it is below $\sim 63$ in coesite-III.

Although penta-coordinated silicon was previously observed in silicates ${ }^{20-24}$, coesite-IV and coesite- $\mathrm{V}$ are the first experimentally observed silica polymorphs featuring $\mathrm{SiO}_{5}$ polyhedra. There are two crystallographically distinctive penta-coordinated silicon atoms ( $\mathrm{Si} 4$ and $\mathrm{Si6}$ ) in coesite-IV and three (Si3, Si4, and Si6) in coesite-V (Fig. 2). Although the range of $\mathrm{Si}-\mathrm{O}$ bond lengths and their average are similar for all $\mathrm{SiO}_{5}$ polyhedra, the shape of the polyhedra varies considerably (Fig. 2), accommodating features common for a trigonal bipyramid and for a square pyramid.

The $\mathrm{SiO}_{6}$ octahedra in both coesite-IV and coesite-V demonstrate a considerable variation in the volume (Supplementary Fig. 4a) and Si-O distances (Supplementary Fig. 4b), but they are comparable with those in stishovite, the only known silica phase with hexa-coordinated silicon whose structure has been reliably and systematically studied experimentally at high pressures $^{25}$. However, the distance between atoms Si2 and Si7 is unexpectedly short $(\sim 2.44 \AA)$. It is by $\sim 8 \%$ shorter than could be anticipated for the structure with the hexa-coordinated silicon at $\sim 50 \mathrm{GPa}$ (Supplementary Fig. $4 \mathrm{c}$ ). The reason is the highly unusual type of connection of $(\mathrm{Si} 2) \mathrm{O}_{6}$ and $(\mathrm{Si} 7) \mathrm{O}_{6}$ octahedrathrough the common face (Fig. 2). Such a structural element has been neither experimentally observed for any silicon compounds, nor expected for small and high-valence cations like $\mathrm{Si}^{4+}$.

In coordinated structures, the occurrence of polyhedra sharing edges or faces is only common for large cations, but very rarely seen for high-valence and low-coordinated (with the coordination number six or lower) small cations. This is the main thrust of third Pauling's rule 26,27 , which is especially strict for compounds such as borates, phosphates, and silicates ${ }^{27}$. Coesite-IV and coesite- $\mathrm{V}$ represent the first experimental examples of violation of third Pauling's rule for $\mathrm{SiO}_{6}$ octahedra linked through faces. In fact, structures of coesite-IV and coesite- $\mathrm{V}$ do not obey also fifth Pauling's rule ("the rule of parsimony") ${ }^{26,27}$. This rule suggests that the number of essentially different kinds of constituents forming a crystal structure tends to be small. Although silicates provide many exceptions from the rule (e.g., the structure of $\mathrm{MgSiO}_{3}$ garnet features both silicon tetrahedra and octahedra), all hitherto-known silica polymorphs ${ }^{15,16,28,29}$ (at least 30 different phases) are built either of tetrahedra or octahedra solely. Thus, coesite-IV and coesite- $\mathrm{V}$ are the first experimentally observed silica polymorphs built of polyhedra of different types.

It should be noted that the complex structure of silica and silicate liquids under compression has been proposed previously. First-principles molecular dynamic simulations on silica liquid ${ }^{7}$ suggest that at high compression, with volume contraction $V / V_{0}$ $<0.7$, which corresponds to compression of coesite beyond 30 $\mathrm{GPa}$ (i.e., exactly the pressure at which a transition to coesite-IV occurs), silicon may simultaneously adopt several types of coordination, namely five-, six-, and sevenfold, with the coordination polyhedra to be significantly distorted. Facesharing of the polyhedra was also suggested, but the kind of the face-sharing was not explicitly specified ${ }^{7}$. Similar results were obtained in molecular dynamic simulations for $\mathrm{MgSiO}_{3}$ glass ${ }^{8}$.

Stability of coesite-IV and coesite-V. A phase with a crystal structure disobeying Pauling's rules is expected to be metastable. $\mathrm{Ab}$ initio simulations (see Methods) confirm that both coesite-IV and coesite- $\mathrm{V}$ are dynamically stable at the pressures where they were observed experimentally (Supplementary Fig. 5). At lower pressure, coesite-IV is more stable than coesite-V (Supplementary Fig. 5, inset), in agreement with the experiment. Calculated equations of state (Supplementary Fig. 6) and lattice parameters
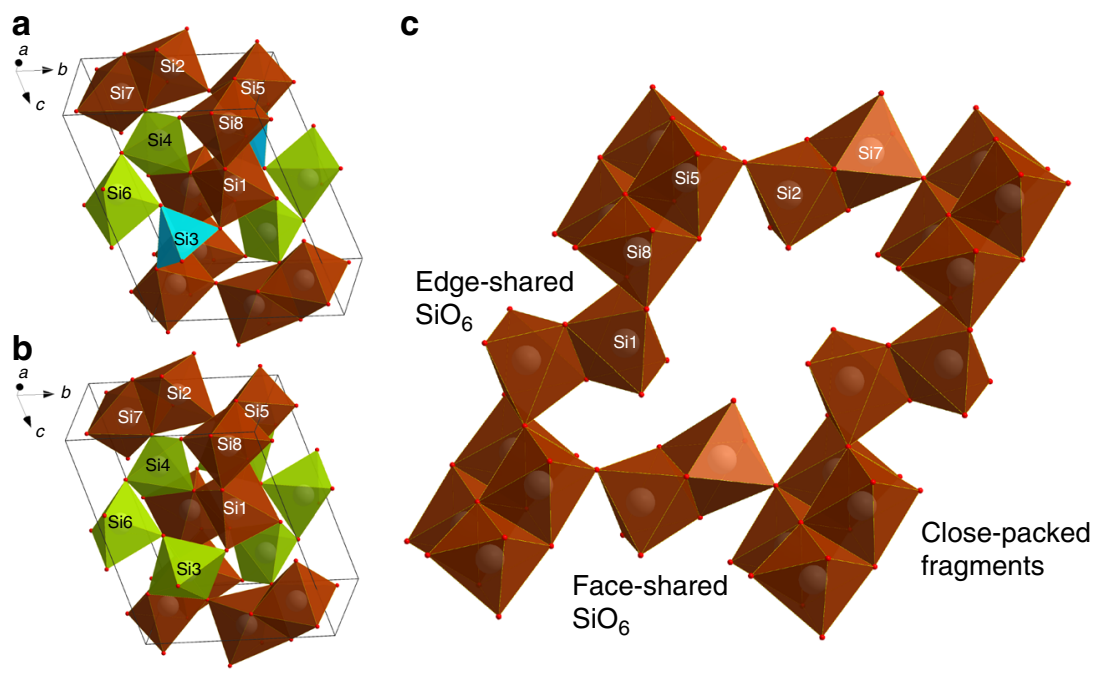

Fig. 2 Crystal structures of coesite-IV and coesite-V. Polyhedral models of the structures of coesite-IV (a) and coesite- $\mathrm{V}$ (b) (SiO 6 octahedra are brown; $\mathrm{SiO}_{5}$ polyhedra are green; $\mathrm{SiO}_{4}$ tetrahedra are blue); a fragment of the structures, similar for both coesite-IV and coesite- $\mathrm{V}$, showing a three-dimensional framework of $\mathrm{SiO}_{6}$ octahedra (c) 
(Supplementary Table 10) of the two phases are in very good agreement with experimental data as well. With increasing pressure, coesite-IV becomes less stable thermodynamically and dynamically, to the extent that it transforms into coesite- $\mathrm{V}$ phase at pressures above $\sim 50 \mathrm{GPa}$ without a barrier (Supplementary Movie 1). At the same time, both phases are energetically highly unfavorable-at $38 \mathrm{GPa}$, where coesite- $\mathrm{V}$ and coesite-IV are nearly degenerate in enthalpy in our theoretical calculations, the calculated enthalpy difference between them and the ground state is $0.389 \mathrm{eV} \cdot$ ion $^{-1}$ (Supplementary Fig. 5, inset). In fact, the very large differences in enthalpy between phases stable at corresponding conditions (stishovite, $\mathrm{CaCl}_{2}$-type, seifertite) and coesite-IV, $\mathrm{V}$ indicate that using modern algorithms of crystal structure predictions or molecular dynamic simulations ${ }^{18,30,31}$, it would be highly difficult (if possible at all) to envisage their existence. On the other hand, the observed enthalpy difference is similar to the values calculated for carbon polymorphs, for example, such as diamond and $\mathrm{C}_{60} 32$.

Notes on the compression mechanism of coesite. All known high-pressure silica phases with six-coordinated silicon are constructed on the basis of distorted hexagonal close-packing (hcp) of oxygen atoms $\mathrm{s}^{27-29,33}$. Pressurization of low-density silica phases (like cristobalite or tridymite), which already contain distorted/defect close-packed oxygen layers ${ }^{29,34}$, results in their transformations into dense phases, built of $\mathrm{SiO}_{6}$-octahedra ${ }^{35,36}$. This happens relatively easily, in a moderate pressure range of $\sim 10-30 \mathrm{GPa}$, as far as within the close-packed oxygen arrays
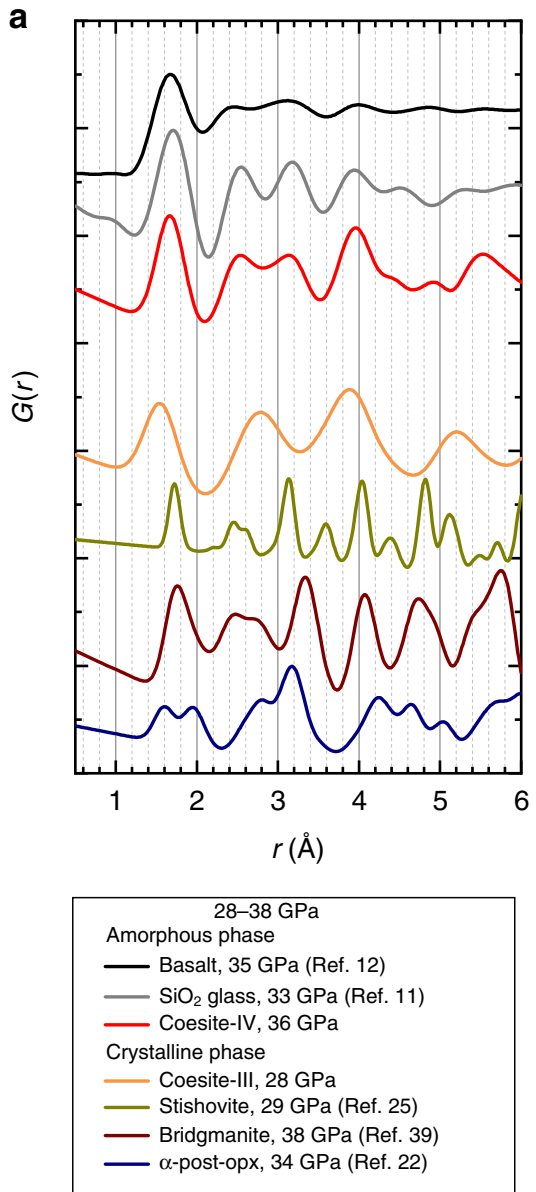

there are only tetrahedral and octahedral interstices, which can be occupied by the cations without severe distortions of the framework. Pressurization of coesite leads to a different result, because its structure does not provide an easy way for the formation of a total hcp framework of oxygen atoms. Although in coesite-IV and coesite- $\mathrm{V}$, one can see fragments closely resembling connections of octahedra in close-packed structures ${ }^{33,34,36}$ (Fig. 2), these fragments do not form continuous layers, and silicon may locate in five-coordinated sites. This picture qualitatively agrees with the changes observed upon the transition from coesite-IV to coesiteV (Fig. 2, Supplementary Fig. 3): ( $\mathrm{Si3}) \mathrm{O}_{4}$-tetrahedra in coesite-IV turn into $(\mathrm{Si} 3) \mathrm{O}_{5}$ polyhedra in coesite- $\mathrm{V}$. It was suggested long time ago and by now generally accepted ${ }^{37}$ that silica and silicate structures are based on close-packing due to a relatively high ionicity of the $\mathrm{Si}-\mathrm{O}$ bond. One could hypothesize that the appearance of an unusual structural element (penta-coordinated silicon, octahedra sharing faces) may be related to increased covalency of $\mathrm{Si}-\mathrm{O}$ bonding with pressure. However, Bader analysis of charge variation in different silica phases with pressure (Supplementary Fig. 7) shows that this is not the case. Our results suggest that penta-coordinated silicon may be a usual component of the intermediate structures or metastable phases upon compression of silicates with oxygen arrays significantly deviated from close-packing (e.g., tectosilicates with large cations, borosilicates, and others).

Comparison with PDFs of silica glass and molten basalt. In Fig. 3 (see also Supplementary Fig. 8), we compare pair-
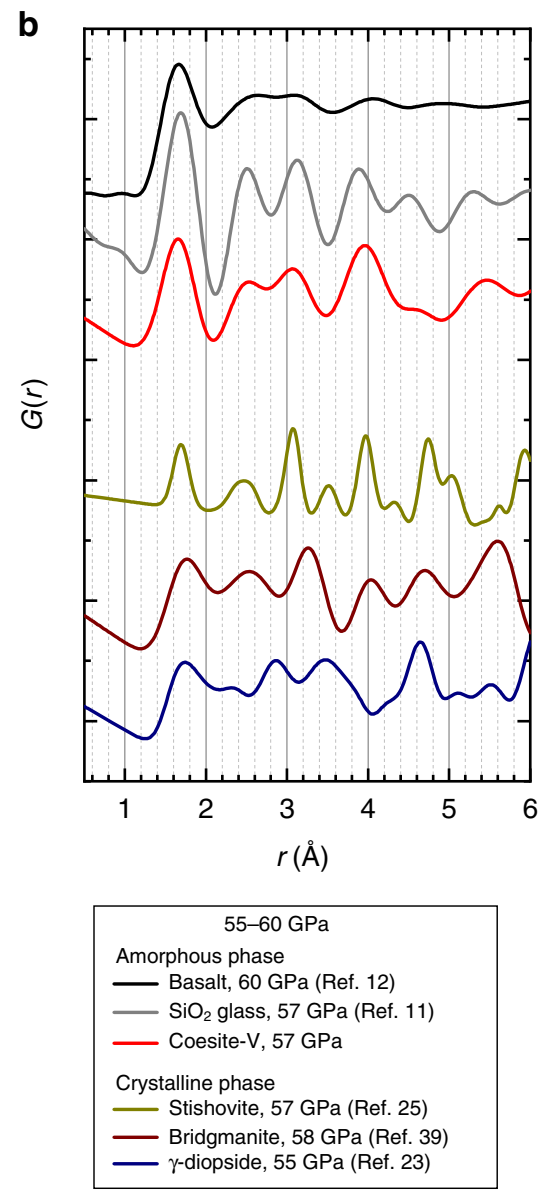

Fig. 3 Pair-distribution functions of silica and silicate phases. Solid curves represent pair-distribution functions calculated for silica polymorphs (this work and ref. ${ }^{25}$ ) and silicates $22,23,39$, compared with those for basalt ${ }^{12}$ and silica ${ }^{11}$ glass measured at different pressures: $\mathbf{a}$, in the range from 28 to $38 \mathrm{GPa} ; \mathbf{b}$, in the range from 55 to $60 \mathrm{GPa}$ 
distribution functions calculated for different silica polymorphs $s^{25,38}$ (including different coesite-derived phases) and silicate $^{22,23,39}$ with the experimental data measured for silica glass $^{11}$ and basalt ${ }^{12}$, as a function of pressure (see Methods). The PDF of silica glass at low pressure has similarities with the PDFs of crystalline silica phases with tetra-coordinated silicon (close positions of the first four peaks), like coesite-I and especially cristobalite. At pressures around or above $30 \mathrm{GPa}$, the silica glass PDF changes considerably; it does not look anymore as from phases containing only $\mathrm{SiO}_{4}$ units (like coesite-III), or only octahedra (like stishovite). On the contrary, there are striking similarities in the PDF of glass at $33 \mathrm{GPa}$ and of coesite-IV at about the same pressure (Fig. 3a). At higher pressures, at about $60 \mathrm{GPa}$, the PDF of glass remains to be alike of the PDF of coesite- $\mathrm{V}$ containing $3 / 8$ of silicon atoms penta-coordinated, and $5 / 8-\mathrm{in} \mathrm{SiO}_{6}$ octahedra (Fig. 3b). The PDFs of molten basalt ${ }^{12}$ at $\sim 30$ and $\sim 60 \mathrm{GPa}$ possess the same features (maxima and minima) as PDFs of coesite-IV and coesite- $\mathrm{V}$ at corresponding pressures. Reported recently ${ }^{40}$, PDFs of $\mathrm{MgSiO}_{3}$ glass up to about $110 \mathrm{GPa}$ are significantly different from that of molten basalt ${ }^{12}$, suggesting that compressed pure $\mathrm{MgSiO}_{3}$ glass cannot represent mantle-related liquids. Densities of coesite-IV and coesite- $\mathrm{V}$ at a pressure above $\sim 45 \mathrm{GPa}$ within the uncertainty of measurements coincide with the density of silica glass ${ }^{10}$ (Supplementary Fig. 9a). These observations are arguments in support of analogous atomic arrangements in compressed silica glass and in coesite-IV or coesite-V. Our results also indicate that (a) silica glass indeed contains $\mathrm{SiO}_{5}$ polyhedra (and not just a mixture of tetrahedra and octahedra in a certain proportion), and (b) in silica glass, a transition from 4- to 6- coordination may not be over at 60-70 $\mathrm{GPa}$, as suggested previously ${ }^{10,41}$.

\section{Discussion}

Our observations imply a possible formation of a very complex network of polyhedra, including face-shared octahedra in silicate liquids at high pressures. A possible influence of such structural peculiarities on the elastic properties of silica (and indirectly on silicate melts) (Supplementary Fig. 9; Supplementary Table 11) may be assessed through a comparison of the compressibility and bulk sound velocities of coesite-IV and coesite- $\mathrm{V}$ with those of dense silica phases with hexa-coordinated silicon (stishovite, $\mathrm{CaCl}_{2}$-structured $\mathrm{SiO}_{2}$, and seifertite) ${ }^{42}$. Obviously, the presence of unconventional structural elements like $\mathrm{SiO}_{5}$ polyhedra and face-sharing octahedra significantly decreases density and, what is even more important, it leads to a drop in the bulk sound velocity above $40 \mathrm{GPa}$ (by about 10\% compared with high-pressure crystalline $\mathrm{SiO}_{2}$ phases; Supplementary Fig. 9c). If silicate liquids with such properties are present in the lower mantle, they should be clearly seismically detectable.

\footnotetext{
Methods

Sample preparation. The starting material for the coesite synthesis was $\mathrm{SiO}_{2}$ glass powder with very low trace-element content, as analyzed at the BGI using LA-ICPMS: Al 20 ppm, Ge 1.3 ppm, Na 1.0 ppm, Li 0.8 ppm, and B, Ti, Fe, Ga, Rb, and Sn below the detection limits. Coesite single crystals were synthesized by mixing the starting powder with $\sim 5 \mathrm{wt} \%$ distilled water inside a platinum capsule, which was then welded shut. The capsule was first placed into pyrophyllite sleeves and then in a $0.5^{\prime \prime}$ talc-pyrex piston-cylinder assembly containing internal, tapered graphite resistance furnaces. The mixture was pressurized to $3.5 \mathrm{GPa}$ and slowly heated up to $1250^{\circ} \mathrm{C}$, kept at this temperature for $\sim 15 \mathrm{~h}$, then cooled down to $1100{ }^{\circ} \mathrm{C}$ in $5 \mathrm{~h}$, and finally quenched. Slow cooling procedure and water-saturated conditions resulted in growth of relatively large (above $100 \mu \mathrm{m}$ in linear dimensions) crystals. No Raman peaks were observed in the spectra of synthesized coesite in the O-H vibration region $\left(2800-3400 \mathrm{~cm}^{-1}\right)$.

Single crystals of coesite with an average size of $0.02 \times 0.02 \times 0.005 \mathrm{~mm}^{3}$ were preselected on a three-circle Bruker diffractometer equipped with a SMART APEX CCD detector and a high-brilliance Rigaku rotating anode (Rotor Flex FR-D, Mo-
} $\mathrm{Ka}$ radiation) with Osmic focusing X-ray optics.
X-ray diffraction. The single-crystal XRD experiments were conducted on the Extreme Conditions Beamline P02.2 at PETRA III, Hamburg, Germany (MAR345dtb image plate detector, Perkin Elmer XRD1621 flat panel detector, $\lambda=$ $0.2898-0.2902 \AA$ ); on the ID09A (now ID15B) beamline at the European Synchrotron Radiation Facility (ESRF), Grenoble, France (MAR555 detector, $\lambda=$ $0.41273 \AA$ ) ; and on the 13-IDD beamline at the Advanced Photon Source (APS), Chicago, USA (MAR165 CCD detector, $\lambda=0.3344 \AA$ ). The X-ray spot size depended on the beamline settings and varied from 4 to $30 \mu \mathrm{m}$. Sample-to-detector distance, coordinates of the beam center, tilt angle, and tilt plane rotation angle of the detector images were calibrated using $\mathrm{CeO}_{2}$ (for data collected at $\mathrm{P} 02.2$ beamline), $\mathrm{Si}$ (ID09A), and $\mathrm{LaB}_{6}$ (13-IDD) powders. XRD images were collected during continuous rotation of DACs typically from -20 to +20 on omega; while data collection experiments were performed by narrow $0.5-1^{\circ}$ scanning of the same omega range. DIOPTAS software ${ }^{43}$ was used for preliminary analysis of the $2 \mathrm{D}$ images and calculation of pressure values from the positions of the XRD lines of $\mathrm{Ne}$

Three sets of experiments were performed. In each experiment, two single crystals of coesite together with a small ruby chip (for pressure estimation) were loaded into BX90-type DACs ${ }^{44}$. Neon was used both as a pressure-transmitting medium and as a pressure standard in all experiments. Neon was loaded with a gasloading system installed at the Bayerisches Geoinstitut ${ }^{45}$.

The first DAC was gradually compressed to $\sim 57 \mathrm{GPa}$, while the single-crystal XRD has been measured only for one crystal at five selected pressure points (namely at 5.8(5), 27.9(5), 35.9(7), 44.5(5), and 57.1(6) GPa). Then, the cell was decompressed with $\sim 5-10 \mathrm{GPa}$ pressure step, and due to sample deterioration, only wide images were measured.

In the second DAC, single-crystal XRD has been measured at six selected pressure points (namely at 14.2(3), 21.6(4), 27.5(3), 29.9(6), 32.7(5), and 43.8(5) GPa) for the first crystal and at two pressure points for another one (27.9(4) and 43.9(4) $\mathrm{GPa}$ ).

In the third run, DAC was compressed to $70 \mathrm{GPa}$, single-crystal XRD has been measured at 5.3(5), 25.6(3), 30.3(6), 36.9(4), 40.3(5), 44.2(4), 49.3(8), 56.8(9), 65.7 (9), and 70.4(9) GPa for the first crystal, and at 5.3(5), 26.3(4), and 30.1(4) GPa for the second one. At selected pressure points, single-crystal XRD was collected in two orientations of the DAC in order to increase data completeness.

It should be noted that only six measurements resulted in successful structure solution and satisfactory refinement (one for coesite-III, four for coesite-IV, and one for coesite-V). The reasons are close peaks overlapping since the samples often contained two phases, early sample deterioration under compression which resulted in peak broadening and low intensity of the reflections.

The detailed summary of the experiments performed, together with determined phase compositions are summarized in Supplementary Table 1. Unit cell parameters of the observed phases are given in Supplementary Table 2. Details of crystal structure refinements of $\mathrm{SiO}_{2}$ high-pressure phases are given in Supplementary Table 3. Supplementary Tables $4-9$ contain information on atomic coordinates and equivalent isotropic displacement parameters of high-pressure coesite phases.

Processing of XRD data (the unit cell determination and integration of the reflection intensities) was performed using CrysAlisPro software ${ }^{46}$. Indexing of the unit cell was performed on about 50 reflections manually selected in the reciprocal space viewer (Ewald explorer implemented in CrysAlisPro software). The reflections were selected in order to follow a 3D lattice in the reciprocal space. Then the found unit cell was refined on the whole set of the reflections with 0.05 tolerance (maximum allowed displacement of the $h, k, l$ indices from an integer). Empirical absorption correction was applied using spherical harmonics, implemented in the SCALE3 ABSPACK scaling algorithm, which is included in the CrysAlisPro software. A single crystal of an orthoenstatite $\left(\left(\mathrm{Mg}_{1.93}, \mathrm{Fe}_{0.06}\right)\left(\mathrm{Si}_{1.93}\right.\right.$, $\left.\mathrm{Al}_{0.06}\right) \mathrm{O}_{6}, P b c a, a=8.8117(2), b=5.18320(10)$, and $\left.c=18.2391(3) \AA\right)$, was used to calibrate the instrument model of CrysAlisPro software (sample-to-detector distance, the detector's origin, offsets of the goniometer angles, and rotation of the $\mathrm{X}$-ray beam and the detector around the instrument axis).

Structure solution and refinement of coesite-III. In the experimental datasets, coesite-III was often found together with either coesite-II or coesite-IV. When coesite-II and coesite-III are found together, orientations of their $\boldsymbol{b}^{*}$ axes coincide, implying that lattice planes $\left(\begin{array}{lll}0 & 1 & 0\end{array}\right)$ of the two crystals should be parallel to each other. In the case of coesite-III and coesite-IV, we observed that the reciprocal plane $a^{*} b^{*}$ of coesite-III coincides with the $a^{*} c^{*}$ plane of coesite-IV. Then the $c$ lattice vector of coesite-III and the $\boldsymbol{b}$ lattice vector of coesite-IV should be colinear.

The crystal structure of coesite-III was refined only at 27.9(5) GPa, due to weakness of the diffraction data in other datasets. At this pressure point, no additional phases (coesite-II or coesite-IV) were found, but instead several domains of coesite-III were observed. The number of overlapped peaks between the most two intense domains did not exceed $20 \%$ of the total peak number. The second domain is rotated relative to the first one by $\sim 178.8^{\circ}$ about the $\boldsymbol{b}^{*}$ axis. The crystal structure of coesite-III was solved using the data from the most intense domain.

In the experiment, we collected 1295 reflections, which were merged based on the crystal symmetry to 833 independent reflections with $R_{\text {int }}=3.29 \%$. At $d=0.8$ $\AA$, completeness of the data was $25.3 \%$. The structure was determined by a direct method using SHELXS ${ }^{47}$ software. After the structure solution, most of the atoms 
were found and the rest of them were located from a series of difference Fourier map cycles. The crystal structure was refined against $F^{2}$ on all data by full-matrix least squares with the SHELXL ${ }^{47}$ software. The amount of the collected data allowed us to refine the structure only in an isotropic approximation. The resulting ratio between data (833) and the number of parameters (143) was $\sim 6$. Five reflections were omitted from the refinement due to their overlap with diamond peaks. The final structure converged with $R_{1}=14.81 \%, w R_{2}=36.32 \%$, and GOF $=$ 1.625 for all 833 unique reflections $\left[R_{1}=13.64 \%, w R_{2}=34.72 \%\right.$, for those 681 data with $I>2 \sigma(I)]$. On a final difference Fourier map, we observed no peaks higher than $1.4 \mathrm{e} \cdot \AA^{-2}$. The most of the intense peaks were within $1 \AA$ of the Si atoms, but several peaks of about $1 \mathrm{e} \cdot \AA^{-2}$ were found nearby oxygen atoms (1.1-1.2 $\AA$ ) probably due to the twinning present. Values of thermal parameters of all atoms except $\mathrm{O}(5)$ are within the normal interval $\left(0.015-0.027 \mathrm{~A}^{2}\right)$. Atom $\mathrm{O}(5)$ is located on a special position $\left(\begin{array}{lll}0 & 0.5\end{array}\right)$ and has a larger thermal parameter $\left(\begin{array}{ll}0.05 \mathrm{~A}^{2}\end{array}\right)$ probably due to the twinning present or even lower symmetry $(P 1)$ of the structure.

Structure solution and refinement of coesite-IV. Coesite-IV has a triclinic symmetry, and data completeness of a single dataset was not enough to solve the crystal structure. In order to increase data completeness, we created a combined reflection file from two $h k l$-files (obtained from datasets collected at 29.9(6) and 40.2(7) GPa) using program XPREP ${ }^{48}$. Then the structure could be successfully determined by the direct method using SHELXS ${ }^{47}$ software. After the structure solution, most of the atoms were found and the rest of them were located from a series of difference Fourier map cycles. The obtained model was used for the refinement of the structure in the individual datasets. The crystal structure was refined against $F^{2}$ on all data by full-matrix least squares with the SHELXL ${ }^{47}$ software. The crystal structure of coesite-IV was refined at 35.9(7), 40.2(7), 44.2(4), and 49.3(8) GPa. At the other pressure points, the structure refinement was not possible due to weakness of the diffraction data.

In the experiment at 35.9(7) $\mathrm{GPa}$, we collected 678 reflections, which were merged based on the crystal symmetry to 452 independent reflections with $R_{\text {int }}=$ 5.49. At $d=0.8 \AA$, completeness of the data was $25.5 \%$. The amount of the collected data allowed us to refine the structure only in an isotropic approximation. The resulting ratio of the number of reflections (452) and the number of parameters (97) was $\sim 5$. Two reflections were omitted from the refinement due to overlap with the diamond peaks. The final structure was refined to convergence with $R_{1}=10.86 \%, w R_{2}=22.80 \%$, and $\mathrm{GOF}=1.122$ for all 452 unique reflections $\left[R_{1}=8.11 \%, w R_{2}=20.83 \%\right.$, for those 332 data with $\left.I>2 \sigma(I)\right]$. On a final difference Fourier map, we observed no peaks higher than $0.7 \mathrm{e} \cdot \AA^{-2}$. The most of the intense peaks were within $1 \AA$ of the $\mathrm{Si}$ atoms. Values of thermal parameters of all atoms are within the normal interval $\left(0.007-0.015 \mathrm{~A}^{2}\right)$

At 40.2(7) GPa, in addition to the standard data collection, we collected the diffraction when DAC was rotated around the X-ray beam direction by $90^{\circ}$. After the integration procedure, two reflection files were combined using program $\mathrm{XRPEP}^{48}$ in order to increase data completeness and redundancy. In total, we collected 2051 reflections, which were merged based upon identical indices to 900 independent reflections with $R_{\text {int }}=7.42 \%$. At $d=0.8 \AA$ completeness of the data was $42.1 \%$. The amount of the collected data allowed us to refine the structure only in an isotropic approximation. The resulting ratio between data (900) and the number of parameters (97) was $\sim 9$. Six reflections were omitted from the refinement due to overlap with diamond peaks. The final structure was refined to convergence with $R_{1}=9.61 \%, w R_{2}=25.99 \%$, and $\mathrm{GOF}=1.109$ for all 900 unique reflections $\left[R_{1}=8.84 \%, w R_{2}=24.76 \%\right.$, for those 789 data with $\left.I>2 \sigma(I)\right]$. On a final difference Fourier map, we observed no peaks higher than $1.4 \mathrm{e} \cdot \AA^{-2}$. The most of the intense peaks were within $1 \AA$ of the Si atoms. Values of thermal parameters of all atoms are within the normal interval $\left(0.016-0.021 \mathrm{~A}^{2}\right)$.

At 44.2(4) GPa, we collected 2963 reflections in total, which were merged based upon identical indices to 2014 independent reflections with $R_{\text {int }}=3.95 \%$. At $d=$ $0.8 \AA$, completeness of the data was $37.0 \%$. The amount of the collected data allowed us to refine the thermal parameters of silicon atoms in an anisotropic approximation, while we could refine those of oxygen atoms in an isotropic approximation. The resulting ratio between data (2014) and the number of parameters (137) was $\sim 15$. Nineteen reflections were omitted from the refinement due to overlap with diamond. The final structure was refined to convergence with $R_{1}=7.41 \%, w R_{2}=13.99 \%$, and GOF $=1.167$ for all 2014 unique reflections $\left[R_{1}=\right.$ $5.55 \%, w R_{2}=12.77 \%$, for those 1562 data with $\left.I>2 \sigma(I)\right]$. On a final difference Fourier map, we observed no peaks higher than $0.8 \mathrm{e} \cdot \AA^{-2}$. The most of the intense peaks were within $1 \AA$ of the Si atoms. Values of thermal parameters of all atoms are within the normal interval $\left(0.006-0.01 \mathrm{~A}^{2}\right)$.

At 49.3(8) GPa, we collected 1524 reflections in total, which were merged based upon identical indices to 968 independent reflections with $R_{\text {int }}=3.97 \%$. At $d=0.8$ $\AA$, completeness of the data was $36.6 \%$. The amount of the collected data allowed us to refine the thermal parameters of all atoms in an isotropic approximation. The resulting ratio between data (968) and the number of parameters (97) was $\sim 10$. Nine reflections were omitted from the refinement due to overlap with diamond. The final structure was refined to convergence with $R_{1}=7.59 \%, w R_{2}=17.40 \%$, and $\mathrm{GOF}=1.124$ for all 968 unique reflections $\left[R_{1}=6.28 \%, w R_{2}=16.35 \%\right.$, for those 798 data with $I>2 \sigma(I)]$. On a final difference Fourier map, we observed no peaks higher than $0.8 \mathrm{e} \cdot \AA^{-2}$. The most of the intense peaks were within $1 \AA$ of the $\mathrm{Si}$ atoms. Values of thermal parameters of all atoms are within the normal interval (0.011-0.016 $\left.\mathrm{A}^{2}\right)$.
Structure solution and refinement of coesite- $\mathbf{V}$. At a single pressure point at 44.5(5) GPa, we observed the coexistence of coesite-IV and coesite-V. The orientations of the crystals were found to be very similar. Due to a small difference in the unit cell volume of coesite-IV and coesite-V, the reflections with the same $h \mathrm{kl}$ indices belonging to two phases appeared to be close to each other.

The crystal structure of coesite- $\mathrm{V}$ was determined only at one pressure point at 56.7(9) GPa, where no admixture of coesite-IV was present. At higher pressures, the amount of the reflection peaks was not enough to refine the crystal structure properly, since the crystals tend to amorphize and few high-angle diffraction peaks could be observed.

In the experiment, we collected 850 reflections, which were merged based upon identical indices to 672 independent reflections with $R_{\text {int }}=5.08 \%$. At $d=0.8 \AA$, completeness of the data was $30.8 \%$. The crystal structure of coesite-IV was used as a starting model for the refinement. The crystal structure was refined against $F^{2}$ on all data by full-matrix least squares with the SHELXL ${ }^{47}$ software. The amount of the collected data allowed us to refine the structure only in an isotropic approximation. The resulting ratio between data (672) and the number of parameters (97) was $\sim 7$. Five reflections were omitted from the refinement due to overlap with diamond. The final structure was refined to convergence with $R_{1}=$ $8.92 \%, w R_{2}=20.2 \%$, and GOF $=1.044$ for all 672 unique reflections $\left[R_{1}=7.28 \%\right.$, $w R_{2}=18.22 \%$, for those 525 data with $\left.I>2 \sigma(I)\right]$. The resulting final difference Fourier map was featureless; no peaks higher than $0.65 \mathrm{e} \cdot \AA^{-2}$ were observed. Values of thermal parameters of all atoms are within the normal interval $\left(0.018-0.023 \mathrm{~A}^{2}\right)$

Pair-distribution functions. The pair-distribution functions were calculated using structural data (CIFs) of the corresponding crystalline phases and DiffPy software (http://www.diffpy.org/products/pdfgui.html).

Computational details. The calculations were based on the density functional theory (DFT) and performed with the projector-augmented wave (PAW) method ${ }^{49,50}$, as implemented in the Vienna Ab-initio Simulation Package (VASP) 51,52 . The PAW potentials with $3 s$ and $3 p$ electrons of $\mathrm{Si}$ and $2 s$ and $2 p$ electrons of $\mathrm{O}$ treated as valence were used. The AM05 exchange-correlation functional ${ }^{53}$ was chosen. It provides a very good agreement of the calculated structural properties with the experimental data. We have checked, however, that the principal results of our theoretical simulations regarding the dynamical and thermodynamic stability of the studied phases of silica do not depend on the choice of the local or semi-local exchange-correlation functional: they hold in both local density approximation and the generalized gradient approximation. The Brillouin zone integration was performed on the $8 \times 8 \times 8$ MonkhorstPack ${ }^{54}$ grid for stishovite/ $\mathrm{CaCl}_{2}$-type silica and seifertite $\left(\alpha-\mathrm{PbO}_{2}\right.$-type) structures and on the $3 \times 3 \times 3$ grid for coesite-IV and coesite-V. The plane-wave energy cutoff was set to $600 \mathrm{eV}$, i.e., by $50 \%$ higher than the default VASP value. The energies of the fully relaxed structures were used together with their volumes and pressures to calculate the enthalpies via the standard definition. Stishovite is considered as the ground-state structure of $\mathrm{SiO}_{2}$ at a pressure below $35 \mathrm{GPa}$. Assuming the typical accuracy of ab initio calculations of the lattice parameters of $\sim 0.01 \AA$, our simulations show that it spontaneously undergoes a tetragonal transformation into a $\mathrm{CaCl}_{2}$-type structure between 35 and $40 \mathrm{GPa}$, in agreement with earlier theoretical work. All enthalpies were calculated relative to the enthalpy of the ground-state structure at the corresponding pressure (Supplementary Fig. 5). The calculated energies, pressures, and volumes were also used to fit theoretical data using the third-order Birch-Murnaghan equation of state.

Phonon dispersion relations of coesite-IV and coesite- $\mathrm{V}$ phases were calculated at $0 \mathrm{~K}$ in the harmonic approximation, using the small displacement method, as implemented in Phonopy code ${ }^{55}$. Crystal structures of the phases were first fully relaxed with constrained unit cell volumes corresponding to the experimental volumes at the respective pressure. Then a force field was obtained in a $2 \times 2 \times$ 2 supercell (384 atoms), based on 144 single atomic displacements with an amplitude of $0.01 \AA$. As our main task was to investigate the dynamic stability/ instability of the new silica phases at room temperature, we did not take into account the LO-TO splitting in high-frequency optical branches. Reciprocal space was sampled using the $2 \times 2 \times 2 \Gamma$-centered $k$-point mesh.

Bonding was characterized by the Bader analysis ${ }^{56}$, which defines that the electronic charge belongs to the particular atom, if it is encountered inside the socalled Bader volumes enclosed by the zero-flux surfaces perpendicular to the minima of the charge density. The so-obtained Bader charge is considered as a good approximation for the total all-electron charge of the corresponding atom.

Computational results. The calculated crystal structure parameters of coesite-IV and coesite- $\mathrm{V}$ phases of $\mathrm{SiO}_{2}$ are presented in Supplementary Table 10. Very good agreement between theoretical and experimental data confirms the reliability of the adopted theoretical approximations for the simulation presented in this work.

Vibrational spectra of coesite-IV at pressure $P=39 \mathrm{GPa}$ and coesite-V at $P=$ $57 \mathrm{GPa}$ are shown in Supplementary Fig. 10a, b, respectively. No imaginary frequencies were observed in either of the phases, i.e., both of them are dynamically stable at pressures where they are observed experimentally. However, our results suggest that coesite-IV and coesite- $\mathrm{V}$ are metastable phases of silica. 
Supplementary Fig. 5 shows the differences between the enthalpies of $\mathrm{SiO}_{2}$ phases considered in this study, coesite-IV, coesite- $\mathrm{V}$, as well as seifertite ( $\alpha$ - $\mathrm{PbO}_{2}$-type structure) relative to the enthalpy of stishovite/ $\mathrm{CaCl}_{2}$-type silica. One can see that the former two are much higher in enthalpy in comparison to the latter. At the same time, the stability of the coesite-IV phase relative to the coesite- $\mathrm{V}$ phase is correctly reproduced in our calculations. Indeed, the former is more stable at lower pressure, while the latter has a lower enthalpy above $38 \mathrm{GPa}$. Note that the optimization (relaxation) of the crystal structure of coesite-IV at unit cell volume of $352.35 \AA^{3}$ resulted in its spontaneous transformation into coesite- $\mathrm{V}$ phase. At this volume (corresponding to experimental pressure of $49 \mathrm{GPa}$, i.e., the highest experimental pressure at which coesite-IV phase is observed experimentally) as well as at lower volumes (higher pressures), coesite-IV phase exhibits instability resulting in coesite-IV $>$ coesite- $\mathrm{V}$ transition. The direct pathway between the phases is shown in Supplementary Movie 1.

The calculated EOSs of coesite-IV and coesite-V phases of $\mathrm{SiO}_{2}$ (Supplementary Fig. 6) show good agreement with the experiment. Calculated bulk moduli and their pressure derivatives are summarized in Supplementary Table 11. In particular, our theoretical results predict that coesite-IV and coesite- $\mathrm{V}$ phases of $\mathrm{SiO}_{2}$ are much softer than stishovite/ $\mathrm{CaCl}_{2}$-type silica and seifertite.

Analyzing the bonding of the investigated silica phases by the Bader analysis (Supplementary Fig. 10), we conclude that the ionicity of the coesite-IV and coesite- $\mathrm{V}$ phases does not change qualitatively, as compared with that of stishovite/ $\mathrm{CaCl}_{2}$ and $\alpha-\mathrm{PbO}_{2}$ phases

\section{Data availability}

The X-ray crystallographic coordinates for structures reported in this article have been deposited at the Inorganic Crystal Structure Database (ICSD) under deposition number CSD (1860556-1860561). These data can be obtained from CCDC's and FIZ Karlsruhe's free service for viewing and retrieving structures (https://www.ccdc.cam.ac.uk/structures/). The crystallographic information (CIFfiles and the corresponding CheckCIF reports) is also available as Supplementary Data 1-12.

Received: 7 May 2018 Accepted: 19 October 2018

Published online: 15 November 2018

\section{References}

1. Nomura, R. et al. Spin crossover and iron-rich silicate melt in the Earth's deep mantle. Nature 473, 199-202 (2011).

2. Stixrude, L. Structure and freezing of $\mathrm{MgSiO}_{3}$ liquid in Earth's lower mantle, Science 310, 297-299 (2005).

3. Labrosse, S., Hernlund, J. W. \& Coltice, N. A crystallizing dense magma ocean at the base of the Earth's mantle. Nature 450, 866-869 (2007).

4. Lee, C.-T. A. et al. Upside-down differentiation and generation of a 'primordial' lower mantle. Nature 463, 930-933 (2010).

5. Stixrude, L., de Koker, N., Sun, N., Mookherjee, M. \& Karki, B. B. Thermodynamics of silicate liquids in the deep Earth. Earth. Planet. Sci. Lett. 278, 226-232 (2009).

6. Petitgirard, S. et al. Fate of $\mathrm{MgSiO}_{3}$ melts at core-mantle boundary conditions. Proc. Natl. Acad. Sci. 112, 14186-14190 (2015).

7. Karki, B. B., Bhattarai, D. \& Stixrude, L. First-principles simulations of liquid silica: structural and dynamical behavior at high pressure. Phys. Rev. BCondens. Matter Mater. Phys. 76, 104205 (2007).

8. Ghosh, D. B., Karki, B. B. \& Stixrude, L. First-principles molecular dynamics simulations of $\mathrm{MgSiO}_{3}$ glass: Structure, density, and elasticity at high pressure. Am. Mineral. 99, 1304-1314 (2014).

9. Du, X. \& Tse, J. S. Oxygen packing fraction and the structure of silicon and germanium oxide glasses. J. Phys. Chem. B 121, 10726-10732 (2017).

10. Petitgirard, $\mathrm{S}$. et al. $\mathrm{SiO}_{2}$ glass density to lower-mantle pressures. Phys. Rev. Lett. 119, 215701 (2017).

11. Prescher, C. et al. Beyond sixfold coordinated $\mathrm{Si}$ in $\mathrm{SiO}_{2}$ glass at ultrahigh pressures. Proc. Natl Acad. Sci. 114, 10041-10046 (2017).

12. Sanloup, C. et al. Structural change in molten basalt at deep mantle conditions. Nature 503, 104-107 (2013).

13. Sato, T. \& Funamori, N. High-pressure structural transformation of $\mathrm{SiO}_{2}$ glass up to 100 GPa. Phys. Rev. B 82, 184102 (2010).

14. Keen, D. A. \& Dove, M. T. Local structures of amorphous and crystalline phases of silica, $\mathrm{SiO}_{2}$, by neutron total scattering. J. Phys. Condens. Matter 11, 9263-9273 (1999).

15. Černok, A. et al. Pressure-induced phase transitions in coesite. Am. Mineral. 99, 755-763 (2014)

16. Černok, A. et al. High-pressure crystal chemistry of coesite-I and its transition to coesite-II. Z. für Krist. —Cryst. Mater. 229, 761-773 (2014).

17. Hu, Q. Y. et al. Polymorphic phase transition mechanism of compressed coesite. Nat. Commun. 6, 6630 (2015).
18. Liu, W. et al. Multiple pathways in pressure-induced phase transition of coesite. Proc. Natl Acad. Sci. 114, 12894-12899 (2017).

19. Robinson, K., Gibbs, G. V. \& Ribbe, P. H. Quadratic elongation: a quantitative measure of distortion in coordination polyhedra. Science 172, 567-570 (1971).

20. Angel, R. J., Ross, N. L., Seifert, F. \& Fliervoet, T. F. Structural characterization of pentacoordinate silicon in a calcium silicate. Nature 384, 441-444 (1996).

21. Finkelstein, G. J., Dera, P. K. \& Duffy, T. S. High-pressure phases of cordierite from single-crystal X-ray diffraction to $15 \mathrm{GPa}$. Am. Mineral. 100, 1821-1833 (2015).

22. Finkelstein, G. J., Dera, P. K. \& Duffy, T. S. Phase transitions in orthopyroxene $\left(\mathrm{En}_{90}\right)$ to $49 \mathrm{GPa}$ from single-crystal X-ray diffraction. Phys. Earth Planet. Inter. 244, 78-86 (2015).

23. Hu, Y., Kiefer, B., Bina, C. R., Zhang, D. \& Dera, P. K. High-pressure $\gamma$ $\mathrm{CaMgSi}_{2} \mathrm{O}_{6}$ : does penta-coordinated silicon exist in the Earth's mantle? Geophys. Res. Lett. 44, 11,340-11,348 (2017).

24. Pakhomova, A. et al. A closer look into close packing: pentacoordinated silicon in a high-pressure polymorph of danburite. IUCrJ 4, 671-677 (2017).

25. Yamanaka, T., Fukuda, T. \& Mimaki, J. Bonding character of $\mathrm{SiO}_{2}$ stishovite under high pressures up to $30 \mathrm{GPa}$. Phys. Chem. Miner. 29, 633-641 (2002).

26. Pauling, $\mathrm{L}$. The principles determining the structure of complex ionic crystals J. Am. Chem. Soc. 51, 1010-1026 (1929).

27. Ulrich, M. Inorganic Structural Chemistry (John Wiley \& Sons Ltd., Chichester, 2006).

28. Haines, J., Léger, J. M., Gorelli, F. \& Hanfland, M. Crystalline post-quartz phase in silica at high pressure. Phys. Rev. Lett. 87, 155503 (2001).

29. Heaney, P. J., Prewitt, C. T. \& Gibbs, G. V. (eds) Silica: Physical Behavior Geochemistry, and Materials Applications. Reviews in Mineralogy Vol. 29 (Mineralogical Society of America, Washington, D.C., 1994).

30. Reilly, A. M. et al. Report on the sixth blind test of organic crystal structure prediction methods. Acta Crystallogr. Sect. B Struct. Sci. Cryst. Eng. Mater. 72, 439-459 (2016)

31. Martoňák, R., Donadio, D., Oganov, A. R. \& Parrinello, M. Crystal structure transformations in $\mathrm{SiO}_{2}$ from classical and ab initio metadynamics. Nat. Mater. 5, 623-626 (2006).

32. Häussermann, U., Simak, S. I., Ahuja, R. \& Johansson, B. Metal-nonmetal transition in the boron group elements. Phys. Rev. Lett. 90, 65701 (2003).

33. Teter, D. M., Hemley, R. J., Kresse, G. \& Hafner, J. High pressure polymorphism in silica. Phys. Rev. Lett. 80, 2145-2148 (1998).

34. Dmitriev, V. P., Tolédano, P., Torgashev, V. I. \& Salje, E. K. H. Theory of reconstructive phase transitions between $\mathrm{SiO}_{2}$ polymorphs. Phys. Rev. B 58, 11911-11921 (1998).

35. Kubo, T., Kato, T., Higo, Y. \& Funakoshi, K.-I. Curious kinetic behavior in silica polymorphs solves seifertite puzzle in shocked meteorite. Sci. Adv. 1, e1500075 (2015).

36. Dubrovinsky, L. et al. A class of new high-pressure silica polymorphs. Phys. Earth Planet. Inter. 143-144, 231-240 (2004).

37. Liebau, F. Pentacoordinate silicon intermediate states during silicate condensation and decondensation. Crystallographic support. Inorg. Chim. Acta 89, 1-7 (1984).

38. Černok, A. et al. Compressional pathways of $\alpha$-cristobalite, structure of cristobalite X-I, and towards the understanding of seifertite formation. Nat. Commun. 8, 15647 (2017)

39. Ballaran, T. B. et al. Effect of chemistry on the compressibility of silicate perovskite in the lower mantle. Earth. Planet. Sci. Lett. 333, 181-190 (2012)

40. Kono, Y., Shibazaki, Y., Kenney-Benson, C., Wang, Y. \& Shen, G. Pressureinduced structural change in $\mathrm{MgSiO}_{3}$ glass at pressures near the Earth's core-mantle boundary. Proc. Natl Acad. Sci. 115, 1742-1747 (2018).

41. Zeidler, A. et al. High-pressure transformation of $\mathrm{SiO}_{2}$ glass from a tetrahedral to an octahedral network: A joint approach using neutron diffraction and molecular dynamics. Phys. Rev. Lett. 113, 135501 (2014).

42. Grocholski, B., Shim, S.-H. \& Prakapenka, V. B. Stability, metastability, and elastic properties of a dense silica polymorph, seifertite. J. Geophys. Res. Solid Earth 118, 4745-4757 (2013).

43. Prescher, C. \& Prakapenka, V. B. DIOPTAS: a program for reduction of twodimensional X-ray diffraction data and data exploration. High. Press. Res. 35, 223-230 (2015).

44. Kantor, I. et al. BX90: a new diamond anvil cell design for X-ray diffraction and optical measurements. Rev. Sci. Instrum. 83, 125102 (2012).

45. Kurnosov, A. et al. A novel gas-loading system for mechanically closing of various types of diamond anvil cells. Rev. Sci. Instrum. 79, 45110 (2008).

46. CrysAlisPro Software system, version 1.171.37.35 (Rigaku Oxford Diffraction, Oxford, UK, 2014).

47. Sheldrick, G. M. A short history of SHELX. Acta Crystallogr. A. 64, 112-122 (2008).

48. XPREP, version 6.12 (Bruker AXS Inc, Madison, Wisconsin, USA, 2001).

49. Blöchl, P. E. Projector augmented-wave method. Phys. Rev. B 50, 17953-17979 (1994).

50. Kresse, G. From ultrasoft pseudopotentials to the projector augmented-wave method. Phys. Rev. B 59, 1758-1775 (1999). 
51. Kresse, G. \& Hafner, J. Ab initio molecular dynamics for liquid metals. Phys. Rev. B 47, 558-561 (1993).

52. Kresse, G. \& Furthmüller, J. Efficiency of ab-initio total energy calculations for metals and semiconductors using a plane-wave basis set. Comput. Mater. Sci. 6, 15-50 (1996).

53. Armiento, R. \& Mattsson, A. E. Functional designed to include surface effects in self-consistent density functional theory. Phys. Rev. B 72, 85108 (2005).

54. Monkhorst, H. J. \& Pack, J. D. Special points for Brillouin-zone integrations. Phys. Rev. B 13, 5188-5192 (1976).

55. Togo, A. \& Tanaka, I. First principles phonon calculations in materials science. Scr. Mater. 108, 1-5 (2015).

56. Henkelman, G., Arnaldsson, A. \& Jónsson, H. A fast and robust algorithm for Bader decomposition of charge density. Comput. Mater. Sci. 36, 354-360 (2006).

\section{Acknowledgements}

N.D. and L.D. thank the German Research Foundation [Deutsche Forschungsgemeinschaft (DFG)] and the Federal Ministry of Education and Research [Bundesministerium für Bildung und Forschung (BMBF), Germany] for financial sup port: projects No. DU 954-11/1, No. DU 393-9/2, and No. DU 393-10/1 (DFG) and Grant No. 5K16WC1 (BMBF). Portions of this work were performed at GeoSoilEnviroCARS (The University of Chicago, Sector 13), Advanced Photon Source (APS), Argonne National Laboratory. GeoSoilEnviroCARS is supported by the National Science Foundation-Earth Sciences (EAR-1634415) and Department of Energy- GeoSciences (DE-FG02-94ER14466). This research used resources of the Advanced Photon Source, a U.S. Department of Energy (DOE) Office of Science User Facility operated for the DOE Office of Science by Argonne National Laboratory under Contract No. DE-AC0206CH11357. A.C. acknowledges support by The Elite Network of Bavaria through the program Oxides. I.A.A., J.T., O.H., and S.I.S. are grateful to the support provided by the Swedish Research Council projects No 2015-04391, 2014-4750, and 637-2013-7296. Support from the Swedish Government Strategic Research Areas in Materials Science on Functional Materials at Linköping University (Faculty Grant SFO-Mat-LiU No 200900971) and the Swedish e-Science Research Centre (SeRC) is gratefully acknowledged. Theoretical analysis of structural properties was supported by the Ministry of Education and Science of the Russian Federation (Grant No. 14.Y26.31.0005). Simulations of the lattice vibrations were supported by the Ministry of Education and Science of the Russian Federation in the framework of Increase Competitiveness Program of NUST "MISIS" (No. K2-2017-080) implemented by a governmental decree dated 16 March 2013, No. 211. Calculations have been carried out at the Swedish National Infrastructure for Computing (SNIC) and at the computer cluster at NUST "MISIS".

\section{Author contributions}

L.D. and N.D. proposed the research and did the project planning. A.C. provided the samples. E.B., A.C., and M.B. selected the single crystals. A.C., E.B., M.B., and L.D prepared the high-pressure experiments. E.B., M.B., A.C., L.D., H.-P.L., M.H., V.P., and C.P., conducted single-crystal X-ray diffraction experiments. E.B. and M.B. analyzed the single-crystal X-ray diffraction data. I.A.A. planned and supervised theoretical calculations. J.T., O.H., S.I.S., M.Be., and I.A.A. conducted ab initio calculations. E.B., M.B., L.D. N.D., and I.A.A. interpreted the results. E.B. and L.D. wrote the paper with contributions of all authors.

\section{Additional information}

Supplementary Information accompanies this paper at https://doi.org/10.1038/s41467018-07265-z.

Competing interests: The authors declare no competing interests.

Reprints and permission information is available online at http://npg.nature.com/ reprintsandpermissions/

Publisher's note: Springer Nature remains neutral with regard to jurisdictional claims in published maps and institutional affiliations.

\begin{abstract}
(c) (P)
Open Access This article is licensed under a Creative Commons Attribution 4.0 International License, which permits use, sharing, adaptation, distribution and reproduction in any medium or format, as long as you give appropriate credit to the original author(s) and the source, provide a link to the Creative Commons license, and indicate if changes were made. The images or other third party material in this article are included in the article's Creative Commons license, unless indicated otherwise in a credit line to the material. If material is not included in the article's Creative Commons license and your intended use is not permitted by statutory regulation or exceeds the permitted use, you will need to obtain permission directly from the copyright holder. To view a copy of this license, visit http://creativecommons.org/ licenses/by/4.0/.
\end{abstract}

(C) The Author(s) 2018 